\title{
Social Accounting and the Public Sector*
}

\author{
Thomas Aronsson \\ Department of Economics, Umeå University \\ SE - 90187 Umeå, Sweden
}

November 2004

\begin{abstract}
This paper contributes to the theory of social accounting. As such, it tries to extend earlier literature on the welfare equivalence of the comprehensive net national product in two main directions, both of which refer to the public sector. One is by considering welfare measurement problems associated with redistributive policy and public good provision, when the public revenues are raised by distortionary taxes. The other is by addressing the consequences of a 'federationlike' decision structure, where independent tax and expenditure decisions are made both by the central government and by lower level governments. In particular, the analysis shows how so called vertical fiscal external effects, which are associated with tax base sharing among the central and lower level governments, contribute to social accounting.
\end{abstract}

Keywords: Welfare measurement, second best, public goods, economic federations.

JEL classification: H21, H23, H41, H70, I31.

*The author would like to thank Karl-Gustaf Löfgren, Tomas Sjögren and Magnus Wikström for helpful comment and suggestions on an earlier version of this paper. A research grant from FORMAS is also gratefully acknowledged. 


\section{Introduction}

During the last three decades, a theory of social accounting has gradually developed. One of the basic ideas behind the study of social accounting is to construct a comprehensive net national product (NNP) measure, which can be used for the purpose of measuring welfare in a dynamic economy. A comprehensive NNP concept is meant to imply an extension of the conventional NNP, such that the extension reflects all relevant aspects of consumption and capital formation for society. The seminal contribution is Weitzman (1976) showing that in a first best setting with a stationary technology, the comprehensive NNP, measured in terms of utility, provides an exact welfare indicator in the sense of being proportional to the present value of future utility facing the representative consumer. Based on Weitzman, the subsequent literature commonly defines the comprehensive NNP in utility terms by using the current value Hamiltonian of the underlying optimal growth problem ${ }^{1}$. More recently, the literature on social accounting has focused attention on welfare measurement problems associated with imperfect market economies $^{2}$; a research area to which the present paper is closely related. A basic insight here is that the 'Hamiltonian-based' type of welfare measure is not in general correct, if the resource allocation is suboptimal from society's point of view.

This paper contributes to the theory of social accounting by focusing on the public sector. In all developed countries, the public sector plays a crucial role for the allocation of resources by providing public services as well as by redistributing income among individuals and groups. The share of total government outlays in GDP averaged slightly above 40 per cent for the OECD countries during 2002, indicating that public sector activities comprise a considerable share of output. Furthermore, public revenues are

\footnotetext{
${ }^{1}$ See Section 2; the current value Hamiltonian measures the utility value of the current consumption (broadly defined) plus the utility value of the current net investments.

${ }^{2}$ This research is summarized by Aronsson et al. (2004).
} 
typically raised by distortionary taxes; as such, there is an additional cost associated with public expenditures, which is relevant for social accounting. Finally, the structure of public decision-making is, itself, likely to be of importance for welfare measurement, because the strategic interaction between different levels of government gives rise to welfare costs.

Surprisingly, however, the theoretical literature on social accounting has devoted very little attention to the public sector. One exception is Aronsson and Löfgren (1999b) dealing with distributional objectives and redistributive policy. Their results imply that, if the resource allocation is first best, then the current value Hamiltonian of the optimal growth problem (i.e. comprehensive NNP in utility terms) constitutes an exact welfare measure also in an economy with heterogeneous consumers. In other words, consideration for redistribution does not necessarily mean that indicators based on the aggregate NNP concept fail to measure welfare. If, on the other hand, the actual distribution of utility and/or consumption is not the outcome of an optimal policy choice, then it may no longer be possible to measure welfare solely by using the current value Hamiltonian, indicating that failure to reach the distributional objectives influences the welfare interpretation of comprehensive NNP. Another exception is Aronsson (1998) dealing with social accounting in an economy with distortionary labor income taxation. He uses a representative agent model where production gives rise to environmental damage and derives a second best analogue to comprehensive NNP in utility terms. The main purposes behind his paper are to relate the welfare measurement problem to the use of distortionary taxes as well as to address environmental aspects of social accounting in a second best framework.

This paper extends the literature on social accounting in two main directions. The first is by considering welfare measurement problems associated with redistributive policy and public good provision in a dynamic economy, where the public revenues are raised by distortionary taxes. The basic question is how the outcome of these policies affects the second best analogue to 
comprehensive NNP. As such, the paper takes a broader view than Aronsson (1998), where the main focus was on the tax system ${ }^{3}$. The other extension is by addressing the consequences of a 'federation-like' decision-structure, where decisions regarding taxes and public expenditures are made both by a central government and by lower level governments. This part of the paper examines how so called vertical fiscal external effects ${ }^{4}$, which are associated with tax base sharing among the central and lower level governments, contribute to social accounting. Although fiscal external effects have received much attention in other areas of economics, there are (to my knowledge) no previous studies where the welfare measurement problem in a dynamic economy is being connected to a federal public decision-structure.

According to the results to be discussed below, although the current value Hamiltonian associated with the second best problem provides an exact welfare measure in utility terms, it is not directly interpretable as an aggregate NNP concept (or the utility value thereof); it also reflects the outcome of redistributive policy. The results also show how the marginal cost of public funds affects the way in which public goods contribute to the welfare measure. The introduction of a federation-like structure takes the economy beyond the second best and implies that a Hamiltonian-based indicator does not necessarily provide an exact welfare measure. Furthermore, the way in which vertical fiscal external effects influence the welfare measure depends on the strategic interaction between the two levels of government. This will be exemplified by considering two possible resource allocations; one in which public policy is determined in the context of a Nash game between the two levels of government, and the other where public policy is determined by a Stackelberg game with the central government acting leader.

\footnotetext{
${ }^{3}$ Although public consumption was part of the model used by Aronsson (1998), its implications for social accounting in a second best economy were never formally addressed.

${ }^{4}$ See the literature on fiscal federalism; e.g. Hansson and Stuart (1987), Johnson (1988), Boadway and Keen (1996), Boadway et al. (1998), Keen and Kotsogiannis (2002) and Dahlby and Wilson (2003).
} 
The outline of the paper is as follows. In Section 2, I present the model and briefly consider welfare measurement in the first best. Although the purpose of the paper is not to measure welfare in a first best equilibrium, this will provide a natural reference case by which to compare the results to be derived later. Section 3 addresses social accounting in a second best economy with only one level of government, whereas a federation structure is introduced in Section 4. Section 5 contains a summary and discussion of the results.

\section{The Model and the First Best Equilibrium}

Since the comprehensive NNP should be designed to reflect all relevant aspects of consumption and capital formation of society, it depends on the structure of preferences and technology. Without loss of generality, and to be able to concentrate on the issues described in the previous section, I disregard the possible influences of environmental and human capital on the preferences and production possibilities ${ }^{5}$. In addition, following most previous studies on social accounting, the population will be assumed to be constant: population growth is not fundamental for the issues discussed below. For the time being, I disregard any distinction between central and local governments by assuming that all policy decisions are made by a central government $^{6}$.

Starting with the preferences, the instantaneous utility function facing consumer $i$ at time $t$ is given by

\footnotetext{
${ }^{5}$ See Hartwick (1990) and Aronsson and Löfgren (1999a) for different aspects of natural and/or environmental resources in the context of social accounting. See Aronsson and Löfgren (1996) for a study of social accounting and human capital.

${ }^{6}$ For the time being, I also abstract from local public goods (i.e. public goods that only benefit the residents of a particular local jurisdiction). I will make a distinction between local and federal public goods in Section 4 .
} 


$$
U^{i}(t)=u\left(c^{i}(t), z^{i}(t)\right)+\Phi(G(t))
$$

for $i=1, \ldots, n$, where $c$ is consumption of a privately provided commodity, $z$ leisure and $G$ the consumption of a public good. Leisure is, in turn, defined as a time endowment, $H$, less the time in market work, $l$. I assume that the functions $u(\cdot)$ and $\Phi(\cdot)$ are increasing in their arguments and strictly concave. The consumers differ with respect to their initial capital stocks, $k^{1}(0), \ldots, k^{n}(0)$, which are exogenously given. The assumption that the public good is additively separable in terms of the utility function is made for expositional ease, although it is in line with the literature on fiscal federalism referred to in the introduction.

Net output is produced by labor and capital according to a neoclassical production function with constant returns to scale, $f(L(t), K(t))$, where $L=\sum_{i} l^{i}$ is the aggregate labor input and $K=\sum_{i} k^{i}$ the aggregate capital stock. Net investments are determined by

$$
\dot{K}(t)=f(L(t), K(t))-C(t)-\rho G(t)
$$

where $C=\sum_{i} c^{i}$, and $\rho$ is a fixed unit cost associated with the provision of the public good (interpretable as the marginal rate of transformation between the public good and the private consumption good).

I begin by briefly recapitulating the welfare measurement problem in the first best equilibrium. To simplify the analysis as much as possible, suppose that the resource allocation is decided upon by a social planner, who is choosing $c^{1}(t), \ldots, c^{n}(t), l^{1}(t), \ldots, l^{n}(t)$ and $G(t)$ to maximize a social welfare function. I assume that the social welfare function is utilitarian

$$
V(0)=\int_{0}^{\infty} \sum_{i=1}^{n} U^{i}(t) e^{-\theta t} d t=\int_{0}^{\infty}\left[\sum_{i=1}^{n} u\left(c^{i}(t), z^{i}(t)\right)+n \Phi(G(t))\right] e^{-\theta t} d t
$$

The restrictions facing the social planner are given by equation (2), the initial condition $K(0)=K_{0}$ and the terminal condition $\lim _{t \rightarrow \infty} K(t) \geq 0$. 
The parameter $\theta$ represents the rate of time preference.

Instead of a utilitarian social welfare function, another possibility would be to define a general instantaneous welfare function, $\varphi\left(U^{1}, \ldots, U^{n}\right)$, where $\varphi(\cdot)$ is increasing in each argument and concave, and then integrate the discounted sum of instantaneous welfare functions over the planning horizon. This change of assumption would necessitate a slightly more burdensome notation; it is not of major importance for the qualitative results derived below. As a consequence, I have chosen the simpler utilitarian objective.

Let $\left\{c^{1, *}(t), \ldots, c^{n, *}(t), l^{1, *}(t), \ldots, l^{n, *}(t), G^{*}(t), \lambda^{*}(t)\right\}_{0}^{\infty}$ solve the social planner's problem, where the superindex "*" is used to denote the first best equilibrium, while $\lambda$ denotes the shadow price of capital. The procedure for measuring welfare in the first best is well established in previous studies. Since the resource allocation is first best, and the optimal control problem time autonomous (except for the nonautonomous time dependence associated with the utility discount factor), it is straight forward to show that the equilibrium satisfies

$$
\theta V^{*}(t)=\sum_{i=1}^{n} u\left(c^{i, *}(t), z^{i, *}(t)\right)+n \Phi\left(G^{*}(t)\right)+\lambda^{*}(t) \dot{K}^{*}(t)
$$

for all $t$, where

$$
V^{*}(t)=\int_{t}^{\infty}\left[\sum_{i=1}^{n} u\left(c^{i, *}(s), z^{i, *}(s)\right)+\Phi\left(G^{*}(s)\right)\right] e^{-\theta(s-t)} d s
$$

is the optimal value function, while the right hand side of equation (4) is the current value Hamiltonian evaluated at the equilibrium. In terms of the model set out here, equation (4) is analogous to the welfare measure derived by Weitzman (1976). It means that the current value Hamiltonian of the underlying optimal growth problem is proportional to the present value of future utility. The current value Hamiltonian is, in turn, interpretable as a measure of comprehensive NNP in utility terms; it measures the current 'utility consumption' - defined as the instantaneous social welfare associated with the current consumption of $c, z$ and $G$ - plus the utility value of the 
current net investments.

The real comprehensive NNP is embedded in equation (4). To illustrate, I follow the convention in the literature on social accounting and linearize the current value Hamiltonian with respect to $c^{i}, z^{i}$ and $G$ (for $i=1, \ldots, n$ ) at the equilibrium. Since the linearized current value Hamiltonian does not, itself, constitute an exact welfare measure (except in the special case where the instantaneous utility function is linear homogenous), one would also need to define a measure of consumer surplus;

$$
j^{i}=u\left(c^{i}, z^{i}\right)+\Phi(G)-u_{c}\left(c^{i}, z^{i}\right) c^{i}-u_{z}\left(c^{i}, z^{i}\right) z^{i}-\Phi_{G}(G) G
$$

where subindices denote partial derivatives. Then, by recognizing that the equilibrium obeys $u_{c}\left(c^{i}, z^{i}\right)-\lambda=0,-u_{z}\left(c^{i}, z^{i}\right)+\lambda w=0$ and $n \Phi_{G}-\lambda \rho=0$, where $w=f_{L}(L, K)$ is the wage rate, we can rewrite equation (4) as

$$
\theta V^{*}(t)=\lambda^{*}(t)\left[C^{*}(t)+w^{*}(t) Z^{*}(t)+\rho G^{*}(t)+\dot{K}^{*}(t)\right]+J^{*}(t)
$$

where $Z=\sum_{i} z^{i}$ and $J=\sum_{i} j^{i}$. The terms within the square bracket constitute, together, the real comprehensive NNP. For the simple economy set out here, the consumption concept of comprehensive NNP consists of three parts; private consumption, leisure and public consumption, whereas the only capital concept involved refers to physical capital. Equation (5) is interpretable such that welfare in real terms at time $t, V^{*}(t) / \lambda^{*}(t)$, is proportional to the sum of real comprehensive NNP and the real consumer surplus $^{7}$, i.e. this sum constitutes an annuity equivalent of welfare.

\footnotetext{
${ }^{7}$ For a thorough analysis of the role of consumer surplus in the context of social accounting, see Li and Löfgren (2002). Their contribution is to relate the sum of the real comprehensive NNP and the real consumer surplus to a real welfare measure defined as the present value of future consumption in an economy with multiple consumption goods and capital goods. As such, they develop practical procedures for calculating the consumer surplus as well as connect social accounting to price index theory. See also Weitzman (2001, 2003).
} 
Turning to the implications of redistribution and public consumption in the context of real comprehensive NNP, two things are worth noticing. First, although the government has objectives for the distribution of private consumption and leisure among agents, the real comprehensive NNP implicit in equation (5) seems to contain no information about the outcome of redistributive policy. The reason is that the first best equilibrium fully implements the distributional objectives. With the social objective function described above, this means that the marginal utility of consumption is equalized among individuals. Therefore, since the optimal redistribution is already implicit in the aggregate consumption of private goods and leisure, there is no need to add any additional component to equation (5) in order to capture the outcome of redistributive policy ${ }^{8}$. Second, the public good should be valued by its production cost in the context of real comprehensive NNP. This means that the marginal contribution of the public good to real comprehensive NNP is given by the marginal rate of transformation between the public good and the private consumption good.

\section{A Second Best Economy}

Note that the basic accounting rules for the public sector discussed in the previous section tend to coincide with procedures applied in practice; we do not, in general, add information to NNP in order to address redistribu-

\footnotetext{
${ }^{8}$ This conclusion does not depend on the use of a utilitarian social welfare function. With reference to the above discussion about the social objective function, replacing the instantaneous utilitarian objective with the general instantaneous social welfare function, $\varphi\left(U^{1}, \ldots, U^{n}\right)$, means that the first order condition $\partial U^{i} / \partial c^{i}-\lambda=0$ must be replaced by

$$
\frac{\partial \varphi\left(U^{1}, \ldots, U^{n}\right)}{\partial U^{i}} \frac{\partial U^{i}}{\partial c^{i}}-\lambda=0 \text { for } i=1, \ldots, n
$$

implying that $\lambda$ still reflects the social (although not the private) marginal utility of consumption. Therefore, given the appropriate reinterpretation of $\lambda$, this more general model also implies equations (4) and (5); see Aronsson and Löfgren (1999b).
} 
tion, and we normally value public services by their production costs. An interesting question is whether these accounting procedures carry over to a second best framework, where the government redistributes and finances public consumption by using distortionary taxes on labor income and capital income. This question has clear practical relevance, since such taxes are commonly used to raise revenues.

\subsection{The Private Sector}

Each consumer chooses private consumption and hours of work at each point in time to maximize the present value of future utility,

$$
\int_{0}^{\infty}\left[u\left(c^{i}(t), z^{i}(t)\right)+\Phi(G(t))\right] e^{-\theta t} d t
$$

subject to an asset accumulation equation. The consumer holds two assets; physical capital, $k$, and government bonds, $b$. Since there is no uncertainty, these two assets have the same rate of return. Define $a^{i}=k^{i}+b^{i}$. The asset accumulation equation is written $^{9}$

$$
\dot{a}^{i}(t)=\bar{r}(t) a^{i}(t)+\bar{w}(t) l^{i}(t)-c^{i}(t)
$$

with $k^{i}(0)=k_{0}^{i}$ and $b^{i}(0)=b_{0}^{i}$, where $\bar{w}=w(1-\tau)$ is the net wage rate, $\bar{r}=r(1-\pi)$ the net interest rate, $\tau$ the labor income tax rate and $\pi$ the capital income tax rate. I also require that each consumer obeys a so called No Ponzi Game (NPG) condition, which ensures that the present value of the asset at the terminal point is nonnegative.

The private agents treat the policy instruments of the government as exogenous. The first order conditions for the private control variables, $u_{c}\left(c^{i}, z^{i}\right)-\phi^{i}=0$ and $-u_{z}\left(c^{i}, z^{i}\right)+\phi^{i} \bar{w}=0$, can be used to derive

\footnotetext{
${ }^{9}$ One may also incorporate other policy instruments, such as income dependent transfers, into the analysis. As long as the government is not able to carry out perfect redistribution - which I assume here - such extensions do not add to the general results derived below.
} 


$$
\begin{aligned}
l^{i}(t) & =l\left(\bar{w}(t), \phi^{i}(t)\right) \\
c^{i}(t) & =c\left(\bar{w}(t), \phi^{i}(t)\right)
\end{aligned}
$$

where $\phi^{i}$ is the marginal utility of wealth in current value terms facing individual $i$. As such, $\phi^{i}$ satisfies

$$
\dot{\phi}^{i}(t)=\phi^{i}(t)[\theta-\bar{r}(t)]
$$

I assume that the production sector is characterized by identical competitive firms, and the technology is given by the constant returns to scale production function presented above. Given these assumptions, the number of firms is not important for the analysis to be carried out below and will be normalized to one for notational convenience. The firm obeys the standard conditions $f_{L}(L, K)-w=0$ and $f_{K}(L, K)-r=0$ for all $t$.

\subsection{The Government}

In the second best problem, the government will be assumed to act as Stackelberg leader, whereas the private sector acts as follower. This means that the necessary conditions characterizing the private sector appear as restrictions facing the government. By substituting equations (7) and (8) into the instantaneous utility function, the instantaneous indirect utility function of individual $i$ is denoted by (neglecting the time indicator)

$$
v^{i}=v\left(\bar{w}, \phi^{i}\right)+\Phi(G)=u\left(c\left(\bar{w}, \phi^{i}\right), H-l\left(\bar{w}, \phi^{i}\right)\right)+\Phi(G)
$$

Note that the instantaneous indirect utility function is defined conditional on $\phi^{i}$, implying that equation (9), defined for $i=1, \ldots, n$, must be part of the set of restrictions facing the government. The other state variables (in addition to $\left.\phi^{1}, \ldots, \phi^{n}\right)$ are the capital stock, $K$, and the stock of government bonds, $B$. The differential equations for the state variables will be described below. 
The optimal tax and expenditure problem can be written $\operatorname{as}^{10}$;

$$
\underset{\bar{w}(t), \bar{r}(t), G(t)}{\operatorname{Max}} \int_{0}^{\infty}\left[\sum_{i=1}^{n} v\left(\bar{w}(t), \phi^{i}(t)\right)+n \Phi(G(t))\right] e^{-\theta t} d t
$$

subject to equations (7) and (8) as well as subject to ${ }^{11}$

$$
\begin{gathered}
\dot{K}(t)=f(L(t), K(t))-C(t)-\rho G(t) \\
\dot{\phi}^{i}(t)=\phi^{i}(t)[\theta-\bar{r}(t)] \text { for } i=1, \ldots n \\
\dot{B}(t)=\bar{r}(t) B(t)+\rho G(t)+\bar{w}(t) L(t)+\bar{r}(t) K(t)-f(L(t), K(t)) \\
\bar{r}(t) \geq 0
\end{gathered}
$$

where $L=\sum_{i} l^{i}, C=\sum_{i} c^{i}, K=\sum_{i} k^{i}$ and $B=\sum_{i} b^{i}$. In addition to the equations of motion for the state variables, I impose the initial conditions $K(0)=K_{0}$ and $B(0)=B_{0}$, the terminal condition $\lim _{t \rightarrow \infty} K(t) \geq 0$ as well as an NPG condition on $B(t)$. The constraint (15) is a minimum restriction ${ }^{12}$

\footnotetext{
${ }^{10}$ This is basically the optimal tax problem analyzed by Chamely (1986), which is here modified by allowing the consumers to differ with respect to their initial wealth.

${ }^{11}$ Using $\dot{B}=r B+\rho G-\tau w L-\pi r[K+B]$ together with $\bar{w}=w(1-\tau), \bar{r}=r(1-\pi)$ and $f(L, K)-w L-r K=0$ gives equation (14).

${ }^{12} \mathrm{~A}$ potential problem in the analysis to be carried out below is that a nonnegativity constraint may imply that the Hamiltonian is not necessarily continuously differentiable with respect to time. At a switch point, where the nonnegativity constraint either becomes binding or is relaxed, the equations of motion for the shadow prices of private wealth, $\dot{\phi}^{i}(i=1, \ldots, n)$, may jump, and the Hamiltonian is not differentiable at this jump point. Chamley (1986) uses a specific utility function and argues that one such switch point exists, where the capital income tax switches from 1 to 0 (at which it then remains forever). To be able to focus on public sector aspect of social accounting without adding too much complexity, I will disregard the potential problem of switch points in what follows. An alternative would be to approximate the Hamiltonian by a continuously differentiable function, implying that the discontinuities vanish.
} 
on the net interest rate: it is written such that the net interest rate must be nonnegative. This restriction effectively prevents the government from taxing a fixed production factor, which would be equivalent to using lumpsum taxation. In the absence of a lower bound on the net interest rate, and since the capital stock is fixed initially, the optimal policy would be to implement a capital income tax such that the net interest rate becomes negative with an arbitrarily large absolute value during an infinitesimal time interval.

\subsection{Welfare Measurement}

To be able to concentrate on the welfare analysis, I will assume that a unique solution exists for the second best problem set out in the previous subsection. Let

$$
\left\{\bar{w}^{0}(t), \bar{r}^{0}(t), G^{0}(t)\right\}_{0}^{\infty}
$$

be the paths for the government's control variables that solve the optimization problem, where the superindex " 0 " is used to denote the second best equilibrium. Substituting the optimal solution back into the current value Hamiltonian of the social optimization problem, we have (neglecting the time indicator for notational convenience)

$$
H^{0}=\sum_{i=1}^{n} v\left(\bar{w}^{0}, \phi^{i, 0}\right)+n \Phi\left(G^{0}\right)+\sum_{i=1}^{n} \zeta^{i, 0} \dot{\phi}^{i, 0}+\lambda^{0} \dot{K}^{0}+\mu^{0} \dot{B}^{0}
$$

where $\zeta^{1}, \ldots, \zeta^{n}, \lambda$ and $\mu$ are costate variables. Defining the optimal value function as in the previous section

$$
V^{0}(t)=\int_{t}^{\infty}\left[\sum_{i=1}^{n} v\left(\bar{w}^{0}(s), \phi^{i, 0}(s)\right)+n \Phi\left(G^{0}(s)\right)\right] e^{-\theta(s-t)} d s
$$

the following relationship is derived in the Appendix;

$$
\theta V^{0}(t)=H^{0}(t)
$$


Equation (18) is analogous to a result derived by Aronsson (1998), although his study was based on a representative agent framework with labor income taxation as the only tax instrument facing the government. By interpreting $\sum_{i} u\left(c^{i}, z^{i}\right)+n \Phi(G)+\lambda \dot{K}$ as the comprehensive NNP in utility terms (as we did in the previous section), one can see from equation (16) that the second best analogue to comprehensive NNP in utility terms contains two additional components. One is represented by $\sum_{i} \zeta^{i} \dot{\phi}^{i}$, which is due to the restriction on the government following from the private intertemporal necessary condition. This sum reflects the social loss following because the marginal utility of wealth differs from the shadow price of physical capital in the second best model. The other is the marginal utility value of government bonds times the accumulation of government bonds, which arises because the stock of government bonds is a state variable in the second best problem.

The main purpose here is to analyze how redistribution and provision of public goods contribute to social accounting in a second best setting. This information is hidden in equation (18). For purposes of comparison with equation (5), it is convenient to define the current value Hamiltonian in terms of the direct instantaneous utility function, and then linearize the instantaneous social welfare function with respect to $c^{i}, z^{i}$ and $G$ (for $i=$ $1, \ldots, n)$. First, note that

$$
v\left(\bar{w}^{0}, \phi^{i, 0}\right)=u\left(c^{i, 0}, z^{i, 0}\right)
$$

where $c^{i, 0}=c\left(\bar{w}^{0}, \phi^{i, 0}\right)$ and $z^{i, 0}=H-l\left(\bar{w}^{0}, \phi^{i, 0}\right)$. Then, define the consumer surplus in the same way as above,

$$
j^{i, 0}=u\left(c^{i, 0}, z^{i, 0}\right)+\Phi\left(G^{0}\right)-u_{c}\left(c^{i, 0}, z^{i, 0}\right) c^{i, 0}-u_{z}\left(c^{i, 0}, z^{i, 0}\right) z^{i, 0}-\Phi_{G}\left(G^{0}\right) G^{0}
$$

with $J^{0}=\sum_{i} j^{i, 0}$. Finally, using the short notations

$$
\phi_{a}^{0}=(1 / n) \sum_{i} \phi^{i, 0}, c_{a}^{0}=(1 / n) \sum_{i} c^{i, 0} \text { and } z_{a}^{0}=(1 / n) \sum_{i} z^{i, 0}
$$


to represent the average marginal utility of wealth ${ }^{13}$, the average private consumption and the average time spent on leisure, respectively, one can derive the following result;

Proposition 1 If the resource allocation is second best, the national welfare measure can be written as

$$
\begin{aligned}
\theta V^{0}= & \phi_{a}^{0}\left[\left(1+\delta_{c}^{0}\right) C^{0}+\bar{w}^{0}\left(1+\delta_{z}^{0}\right) Z^{0}+\rho\left(1+\beta^{0}\right) G^{0}\right] \\
& +\sum_{i=1}^{n} \zeta^{i, 0} \dot{\phi}^{i, 0}+\lambda^{0} \dot{K}^{0}+\mu^{0} \dot{B}^{0}+J^{0}
\end{aligned}
$$

where $1+\beta^{0}=1+\left(\lambda^{0}-\phi_{a}^{0}-\mu^{0}\right) / \phi_{a}^{0}, \delta_{c}^{0}=\operatorname{cov}\left(\phi^{0}, c^{0}\right) /\left(\phi_{a}^{0} c_{a}^{0}\right)$ and $\delta_{z}^{0}=$ $\operatorname{cov}\left(\phi^{0}, z^{0}\right) /\left(\phi_{a}^{0} z_{a}^{0}\right)$.

Proof: See the Appendix.

To shorten the notations, the time indicator has been dropped in the welfare measure described in Proposition 1. As before, the sum of the linearized current value Hamiltonian and the consumer surplus constitutes an exact welfare measure. Here, one may interpret the linearized current value Hamiltonian as a second best analogue to real comprehensive NNP multiplied by the average marginal utility of consumption. Therefore, denoting the second best analogue to real comprehensive NNP by $R^{0}$, the welfare measure in Proposition 1 can also be written as $\theta V^{0}(t)=\phi_{a}^{0}(t) R^{0}(t)+J^{0}(t)$.

By comparing the formula in the proposition with equation (5), it follows that the linearized current value Hamiltonian associated with the second best problem takes a different form than its first best counterpart. Notice that the 'consumption part', i.e. the first row of the formula in the proposition, does not only contain the aggregate variables $C, Z$ and $G$ (as it did in the first best framework in Section 2); the distribution of private consumption and leisure among consumers also affects the welfare measure. This is seen

\footnotetext{
${ }^{13}$ Since $u_{c}\left(c^{i}, z^{i}\right)-\phi^{i}=0$ for $i=1, \ldots, n$, 'marginal utility of consumption' and 'marginal utility of wealth' will be used synonymously.
} 
by the appearance of the distributional characteristics ${ }^{14}$ for consumption and leisure, $\delta_{c}^{0}$ and $\delta_{z}^{0}$. The intuition is that the government is not able, in this case, to equalize the marginal utility of consumption among consumers, implying that it is not able to fully implement its distributional objectives. To be able to measure how private consumption and leisure affect the second best analogue to real comprehensive NNP, therefore, it is necessary to add information that reflects the extent to which private consumption and leisure differ among individuals as a consequence of differences in the marginal utility of consumption (i.e. the extent to which the government fails to reach its distributional objectives). This information is summarized by the distributional characteristics ${ }^{15}$. One may also interpret $\left(1+\delta_{c}^{0}\right)$ and $\bar{w}^{0}\left(1+\delta_{z}^{0}\right)$ as representing the 'marginal accounting prices' for aggregate private consumption and aggregate leisure, respectively, in the second best framework analyzed here. The corresponding marginal accounting prices in the first best equilibrium are 1 and $w$, respectively; see Section 2 . In general, $\delta_{c}$ and $\delta_{z}$ can take both positive and negative values along the general equilibrium path; however, if $u_{c z}(c, z) \geq 0$, then $\delta_{c}<0$ and $\delta_{z}<0$. The latter would imply that the second best analogue to real comprehensive NNP attaches lower weight to aggregate private consumption and leisure than does its first best counterpart.

The social accounting rule for the public good also differs from the rule derived in the first best equilibrium. To provide some intuition, note that

\footnotetext{
${ }^{14}$ Note that

$$
\begin{aligned}
\operatorname{cov}\left(\phi^{0}, c^{0}\right) & =\frac{1}{n} \sum_{i=1}^{n} \phi^{i, 0} c^{i, 0}-\phi_{a}^{0} c_{a}^{0} \\
\operatorname{cov}\left(\phi^{0}, z^{0}\right) & =\frac{1}{n} \sum_{i=1}^{n} \phi^{i, 0} z^{i, 0}-\phi_{a}^{0} z_{a}^{0}
\end{aligned}
$$

${ }^{15}$ With a common utility function, the distributional characteristics are (at least in principle) estimable by using econometric methods. An introduction to relevant methods for analyzing consumer demand are described by e.g. Deaton (1986).
} 
the first order condition for $G$ can be written as

$$
n \Phi_{G}\left(G^{0}\right)=\left(\lambda^{0}-\mu^{0}\right) \rho
$$

where $-\mu$ (the negative of the shadow price of government bonds) is interpretable as the marginal excess burden in utility terms; so, $\lambda-\mu$ measures the marginal cost of public funds in utility terms. The marginal value of $G$ implicit in the second best analogue to real comprehensive NNP is measured by the production cost, $\rho$, times $(1+\beta)$, where the latter is interpretable as the marginal cost of public funds in terms of the average marginal utility of consumption. One may interpret $\rho(1+\beta)$ as the marginal accounting price for the public good in the second best framework, whereas the corresponding marginal accounting price in the first best equilibrium is $\rho$. If $(1+\beta)>1$, as one would normally expect, the marginal accounting price of a public good is typically underestimated in traditional social accounting.

\section{Social Accounting in an Economic Federation}

In the previous section, the public sector was treated as one decision maker. However, real world public sectors are commonly organized in several levels, where each such level is able to make its own tax and expenditure decisions. Different levels of government may also share common tax bases. In this section, I will consider social accounting in a stylized economic federation with two levels of government; local governments and a central government. A basic question is whether or not the current value Hamiltonian underlying the central government's optimization problem constitutes a welfare indicator in the same way as it did in the previous section, where a unified government was assumed. This question has clear practical relevance, since the current value Hamiltonian associated with the central government's policy problem provides the basis for calculating comprehensive NNP for the economy as a whole. Particular attention will be paid to the implications 
of tax base sharing among the two levels of government. The choice of concentrating on vertical fiscal interaction is natural, considering that earlier literature on social accounting does not address the consequences of public decision-making within the framework of a multi-level government.

To be able to concentrate on the implications of tax base sharing, two simplifying assumptions are added. First, I disregard any horizontal interaction among local governments (such as those associated with spillover effects of local public goods and labor mobility). This assumption does not reflect a belief that horizontal external effects are unimportant; only that their qualitative contributions to the welfare measures are similar to those of other external effects already addressed by previous studies. Second, since the consequences for welfare measurement of allowing the consumers to differ with respect to their capital endowments were thoroughly addressed in the previous section, I simplify by assuming that all consumers are equal and normalize the number of residents in each locality to one. The latter is not important for the qualitative results to be derived regarding vertical fiscal interaction.

The local governments as well as the central government are assumed to supply public goods, which are consumed by the residents in their respective jurisdictions, and both levels of governments are assumed to be able to run budget deficits ${ }^{16}$. However, whereas the central government uses taxes on both labor income and capital income (as in the previous section), I restrict the set of tax instruments of the local governments to include only the labor income tax rate ${ }^{17}$. This assumption means that the marginal cost

\footnotetext{
${ }^{16}$ There is a variety of empirical studies on the intertemporal behavior of local governments, where one of the major issues has been to test whether local governments behave in a way that is consistent with intertemporal optimization. The results are mixed; see e.g. Holtz-Eakin et al. (1994), Dahlberg and Lindström (1998) and Tovmo (2004). To make the analysis as general as possible, I have chosen to allow the local governments to run budget deficits. The balanced budget situation appears as a special case of the model and will be discussed.

${ }^{17}$ Several earlier studies on fiscal federalism concentrate on labor income taxation, and
} 
of public funds is likely to differ between the two levels of government, which is arguably realistic. It has no other important implications for the results.

The notations are the same as in the previous section (except that the superindex " $i "$ is dropped for obvious reasons). To be able to distinguish between the public goods provided by different levels of government, the instantaneous utility function facing the consumers will be rewritten as

$$
U=u(c, z)+\Psi(g, G)
$$

where $g$ is a local public good provided by the local government. The function $\Psi(\cdot)$ is increasing in each argument and strictly concave. The consumers treat the policy instruments of the central and local governments as exogenous. In each locality, the consumer chooses his/her consumption of the private good, $c$, and supplies labor, $l$. The private necessary conditions are written as follows;

$$
\begin{aligned}
c(t) & =c(\bar{w}(t), \phi(t)) \\
l(t) & =l(\bar{w}(t), \phi(t)) \\
\dot{\phi}(t) & =\phi(t)[\theta-\bar{r}(t)] \\
\dot{a}(t) & =\bar{r}(t) a(t)+\bar{w}(t) l(t)-c(t)
\end{aligned}
$$

The private asset is here defined as $a=k+b_{l}+b_{c}$, where $b_{l}$ and $b_{c}$ are interpretable in terms of local government debt and central government debt, respectively, at the individual level. In addition, $\bar{w}=w\left(1-\tau_{l}-\tau_{c}\right)$, where $\tau_{l}$ is the local labor income tax rate and $\tau_{c}$ the labor income tax rate of the central government, whereas $\bar{r}=r(1-\pi)$ as in the previous section.

assume that the labor income tax base is shared between the central and lower level governments. In practice, local and regional tax instruments seem to vary considerably across countries, although the sets of tax instruments facing local and regional governments are typically more limited than that facing a central government. Here, it is not of main importance to describe the local set of tax instruments in the most realistic way; only that at least one of the tax bases is shared between the two levels of government. 
Turning, finally, to the production side of the economy, the output in each locality is produced by identical competitive firms, the number of which is normalized to one. The production technology is represented by the production function $f(l, k)$, which has the same properties as in the previous section. To avoid complications, which are not essential for the qualitative results, I disregard trade among localities.

\subsection{The Local Governments}

The levels of the local and national policy variables are assumed to be decided upon before the private agents make their decisions. Each government recognizes, and incorporate into its decision problem, how the private sector responds to the policy variables facing this particular government. I also assume that each local government acts as a Nash competitor towards the other local governments as well as towards the central government.

There are $n$ local governments (as well as residents of the federation as a whole), which are identical by assumption. Each local government chooses the local labor income tax rate, $\tau_{l}$, and the local public $\operatorname{good}^{18}, g$, at each point in time to maximize the present value of future utility facing its resident;

$$
\int_{0}^{\infty}[v(\bar{w}(t), \phi(t))+\Psi(g(t), G(t))] e^{-\theta t} d t
$$

subject to

$$
\begin{gathered}
\dot{k}(t)=f(l(t), k(t))-c(t)-\rho_{l} g(t)-\frac{1}{n} \rho_{c} G(t) \\
\dot{\phi}(t)=\phi(t)[\theta-\bar{r}(t)] \\
\dot{b}_{l}(t)=r(t) b_{l}(t)+\rho_{l} g(t)-\tau_{l}(t) w(t) l(t)
\end{gathered}
$$

\footnotetext{
${ }^{18}$ Note that $g$ is also interpretable as a publicly provided private good.
} 
as well as subject to equations (19) and (20). The factor prices are given by $w=f_{l}(l, k)$ and $r=f_{k}(l, k)$, while the constants $\rho_{l}$ and $\rho_{c}$ are interpretable as the marginal rate of transformation between public and private goods facing the local government and the central government, respectively. In addition, the local government obeys the same type of initial, terminal and NPG conditions as those described in the previous section. Note that the budget constraint contains no intergovernmental grants. Provided that the central government is unable to implement the second best resource allocation $^{19}$, which I will assume throughout this section, this simplification does not affect the qualitative results do be derived below.

The current value Hamiltonian underlying the local government's decision problem is written (neglecting the time indicator for notational convenience)

$$
H_{l}=v(\bar{w}, \phi)+\Psi(g, G)+\zeta_{l} \dot{\phi}+\lambda_{l} \dot{k}+\mu_{l} \dot{b}_{l}
$$

where the subindex " $l$ " indicates 'local government'. Each local government treats the paths of $\tau_{c}, \pi$ and $G$ as exogenous during optimization. By using the first order conditions for the local control variables, $\tau_{l}$ and $g$, we can derive

$$
\begin{aligned}
\tau_{l} & =\tau_{l}\left(k, \phi, \zeta_{l}, \lambda_{l}, \mu_{l}, \tau_{c}, \pi\right) \\
g & =g\left(\lambda_{l}, \mu_{l}, G\right)
\end{aligned}
$$

where the dependence of $\tau_{l}$ and $g$ on constants has been suppressed, whereas the costate variables satisfy

$$
\dot{\zeta}_{l}=\theta \zeta_{l}-\frac{\partial H_{l}}{\partial \phi}
$$

\footnotetext{
${ }^{19}$ If the central government were able to implement the second best resource allocation, then the welfare analysis carried out in the previous section still applies, meaning that the special case of full implementation has already been dealt with.
} 


$$
\begin{aligned}
& \dot{\lambda}_{l}=\theta \lambda_{l}-\frac{\partial H_{l}}{\partial k} \\
& \dot{\mu}_{l}=\theta \mu_{l}-\frac{\partial H_{l}}{\partial b_{l}}
\end{aligned}
$$

I will return to the conditions obeyed by the local public sector below, when a Stackelberg game between the two levels of government is being analyzed.

\subsection{The Central Government as a Nash Competitor}

The central government chooses $\tau_{c}, \pi$ and $G$ to maximize the following utilitarian objective function;

$$
\int_{0}^{\infty} n[v(\bar{w}(t), \phi(t))+\Psi(g(t), G(t))] e^{-\theta t} d t
$$

which is the sum of objectives of the local governments.

The set of constraints perceived by the central government depends on whether or not it recognizes (and incorporates into its decision problem) how the local public sector responds to changes in $\tau_{c}, \pi$ and $G$. Consider first the situation where the central government acts as a Nash competitor ${ }^{20}$ towards the local governments. This situation means that it faces the same types of constraints as it did in the previous section ${ }^{21}$;

$$
\begin{gathered}
\dot{K}(t)=f(L(t), K(t))-C(t)-n \rho_{l} g(t)-\rho_{c} G(t) \\
\dot{\phi}(t)=\phi(t)[\theta-\bar{r}(t)]
\end{gathered}
$$

${ }^{20} \mathrm{~A}$ standard reference to game theory applications in the context of intertemporal optimization problems is Basar and Olsder (1982).

${ }^{21}$ The accumulation equation for central government debt is written such as to emphasize its dependence on $\tau_{l}$ and $B_{l}$. By defining $B=B_{l}+B_{c}$ and using

$$
\dot{B}_{c}=r B_{c}+\rho_{c} G-\tau_{c} w L-\pi r[K+B]
$$

together with $f(L, K)-w L-r K=0$ and $\bar{w}=w\left(1-\tau_{l}-\tau_{c}\right)$, we obtain equation (36). 


$$
\begin{aligned}
\dot{B}_{c}(t)= & \bar{r}(t) B_{c}(t)-[r(t)-\bar{r}(t)] B_{l}(t)+\rho_{c} G(t)+\bar{w}(t) L(t) \\
& +\bar{r}(t) K(t)+\tau_{l}(t) w(t) L(t)-f(L(t), K(t))
\end{aligned}
$$

$$
\bar{r}(t) \geq 0
$$

where $C=n c, L=n l, K=n k, B_{c}=n b_{c}$ and $B_{l}=n b_{l}$.

Suppose that the central government plays a Nash game in open loop form with the local governments. The open loop assumption simplifies the analysis, since it means that the whole control path will be chosen at the outset of the game ${ }^{22}$. It is well known that differential games are very difficult to solve analytically, and that a solution may not exist ${ }^{23}$. However, if it does exist, it has important implications for welfare measurement. To see this, let the equilibrium control path be denoted (assuming it exists) ${ }^{24}$

$$
\left\{\tau_{c}^{\bullet}(t), \pi^{\bullet}(t), G^{\bullet}(t), \tau_{l}^{\bullet}(t), g^{\bullet}(t)\right\}_{0}^{\infty}
$$

The current value Hamiltonian facing the central government, if evaluated at the Nash equilibrium, can be written (neglecting the time indicator)

$$
H_{c}^{\bullet}=n\left[v\left(\bar{w}^{\bullet}, \phi^{\bullet}\right)+\Psi\left(g^{\bullet}, G^{\bullet}\right)\right]+\zeta_{c}^{\bullet} \dot{\phi}^{\bullet}+\lambda_{c}^{\bullet} \dot{K}^{\bullet}+\mu_{c}^{\bullet} \dot{B}_{c}^{\bullet}
$$

\footnotetext{
${ }^{22} \mathrm{An}$ alternative to the open loop assumption would be to formulate the Nash game in feedback loop form. In a different context, Aronsson et al. (2004) show that, although Nash games in open loop and feedback loop forms differ with respect to the set of first order conditions implicit in the resource allocations, the resulting welfare measures have a similar structure. As a consequence, I use the open loop framework here, since it is more convenient from a technical point of view than the Nash game in feedback loop form.

${ }^{23}$ Explicit solutions usually require a set of simplifying assumptions; see e.g. Lancaster (1973) and Hoel (1978). In a more general setting, very few insights emerge (even in terms of qualitative statements).

${ }^{24}$ This means that (i) $\left\{\tau_{c}^{\bullet}(t), \pi^{\bullet}(t), G^{\bullet}(t)\right\}_{0}^{\infty}$ solve the central government's optimization problem conditional on $\tau_{l}(t)=\tau_{l}^{\bullet}(t)$ and $g(t)=g^{\bullet}(t)$ for all $t$, and (ii) $\left\{\tau_{l}^{\bullet}(t), g^{\bullet}(t)\right\}_{0}^{\infty}$ solve the local governments' optimization problems conditional on $\tau_{c}(t)=\tau_{c}^{\bullet}(t), \pi(t)=$ $\pi^{\bullet}(t)$ and $G(t)=G^{\bullet}(t)$ for all $t$.
} 
where the subindex "c" indicates 'central government'. By using the optimal value function,

$$
V^{\bullet}(t)=\int_{t}^{\infty} n\left[v\left(\bar{w}^{\bullet}(s), \phi^{\bullet}(s)\right)+\Psi\left(g^{\bullet}(s), G^{\bullet}(s)\right)\right] e^{-\theta(s-t)} d s,
$$

together with equation (38), while assuming that the present value Hamiltonian, $H_{c}^{\bullet}(t) e^{-\theta t}$, approaches zero when time goes to infinity ${ }^{25}$, one can derive the following result;

Proposition 2If the central government plays a Nash game in open loop form with the local governments, the national welfare measure can be written as

$$
\begin{gathered}
\theta V^{\bullet}(t)=H_{c}^{\bullet}(t)+\int_{t}^{\infty}\left[\mu_{c}^{\bullet}(s)\left\{w^{\bullet}(s) L^{\bullet}(s) \dot{\tau}_{l}^{\bullet}(s)-\pi^{\bullet}(s) r^{\bullet}(s) \dot{B}_{l}^{\bullet}(s)\right\}\right. \\
\left.+\Delta^{\bullet}(s) \dot{g}^{\bullet}(s)\right] e^{-\theta(s-t)} d s
\end{gathered}
$$

where $\Delta^{\bullet}=\partial H_{c}^{\bullet} / \partial g=n\left[\Psi_{g}\left(g^{\bullet}, G^{\bullet}\right)-\rho_{l} \lambda_{c}^{\bullet}\right]$

Proof: see the Appendix.

A general interpretation of the formula in Proposition 2 is that, if the central government behaves as a Nash competitor, then the current value Hamiltonian of the central government's optimization problem does not, in general, constitute an exact welfare indicator. The reason is that the local income tax rate, $\tau_{l}$, the aggregate of local public debt, $B_{l}$, and the local public goods, $g$, are exogenous to the central government. This means that the values of changes in $\tau_{l}, B_{l}$ and $g$ are not internalized by the behavior of the central government and must, therefore, be added to the central government's current value Hamiltonian in order to arrive at an exact welfare

\footnotetext{
${ }^{25}$ Michel (1982) shows that the present value Hamiltonian of a well defined optimal control problem approaches zero when time goes to infinity. He assumes that the only nonautonomous time dependence is associated with the utility discount factor. See Seierstad and Sydsaeter (1987, page 245) for an extension to the situation where the instantaneous utility and/or production function may exhibit an explicit time dependence.
} 
measure. Note also that these additional terms are forward looking. As a consequence, and by contrast to the second best model analyzed in Section 3 , welfare at time $t$ cannot solely be measured by using entities referring to time $t$. The practical implication is, of course, that welfare measurement becomes more difficult, since the value of marginal external effects cannot be directly inferred from market data.

The second part of the first row represents the welfare contributions of the vertical fiscal external effect. To provide further intuition, recall that this external effect is caused by tax base sharing. If $\mu_{c}^{\bullet}<0$, as would normally be expected, one can interpret $\int_{t}^{\infty} \mu_{c} w L \dot{\tau}_{l} \exp (-\theta(s-t)) d s$ to measure the welfare cost (gain) following as $\dot{\tau}_{l}>0(<0)$ increases (decreases) the debt of the central government, ceteris paribus, by decreasing (increasing) the available labor income tax base. Similarly, $-\int_{t}^{\infty} \mu_{c} \pi r \dot{B}_{l} \exp (-\theta(s-t)) d s$ is interpretable as the welfare gain (cost) following as $\dot{B}_{l}>0(<0)$ implies an external increase (decrease) of the capital income tax revenues of the central government. It is important to emphasize that $\dot{\tau}_{l}$ and $\dot{B}_{l}$ can take both positive and negative values along the Nash equilibrium path, implying that the welfare contribution of the second part of the first row can be either positive or negative. In the special case with balanced budgets for the local governments, the welfare measure takes the same general form as in Proposition 2 with the exception that $\dot{B}_{l}=0$ for all $t$. The second row of the formula in the proposition is also interpretable in terms of an external effect, since the local public goods are exogenous to the central government. As such, they contribute to the welfare measure because $\Delta$ is generally nonzero.

There is an interesting special case where the accounting principles corresponding to the second best resource allocation also apply in the present context;

Corollary 1: If $\dot{\tau}_{l}^{\bullet}(t)=0, \dot{B}_{l}^{\bullet}(t)=0$ and $\dot{g}^{\bullet}(t)=0$ for all $t$, social welfare is proportional to the current value Hamiltonian implicit in the central government's optimization problem, i.e. $\theta V^{\bullet}(t)=H_{c}^{\bullet}(t)$. 
Notice that Corollary 1 is not based on the assumption that the fiscal external effects have become internalized. It means, instead, that if the local labor income tax rate, the local public debt and the local public good do not change over time, then the 'exogenous' variables facing the central government remain fixed at their initial values. As such, they will not contribute to welfare other than via the current value Hamiltonian, meaning that the forward looking terms in Proposition 2 vanish from the welfare measure. This implies that the current value Hamiltonian facing the central government is interpretable as a welfare measure in the same general way as in the second best setting.

By analogy to the short notations used in Section 3 , let $1+\beta_{l}=1+$ $\left(\lambda_{l}-\phi-\mu_{l}\right) / \phi$ and $1+\beta_{c}=1+\left(\lambda_{c}-\phi-\mu_{c}\right) / \phi$ represent the marginal cost of public funds facing the local governments and the central government, respectively. Another corollary to Proposition 2 immediately follows;

Corollary 2: The 'marginal accounting prices' for $G$ and $g$ are given by $\rho_{c}\left(1+\beta_{c}\right)$ and $\rho_{l}\left(1+\beta_{l}\right)$, respectively.

Corollary 2 is interesting because the linearized current value Hamiltonian provides the key behind the construction of an analogue to real comprehensive NNP, in which the above accounting prices apply. To explain Corollary 2 more thoroughly, let me once again linearize the current value Hamiltonian and rewrite the welfare measure (neglecting the time indicator)

$$
\begin{aligned}
\theta V^{\bullet}= & \phi^{\bullet}\left[C^{\bullet}+\bar{w}^{\bullet} Z^{\bullet}+p_{l}\left(1+\beta_{l}^{\bullet}\right) n g^{\bullet}+\rho_{c}\left(1+\beta_{c}^{\bullet}\right) G^{\bullet}\right] \\
& +\zeta_{c}^{\bullet} \dot{\phi}^{\bullet}+\lambda_{c}^{\bullet} \dot{K}^{\bullet}+\mu_{c}^{\bullet} \dot{B}_{c}^{\bullet}+J^{\bullet}+\Omega^{\bullet}
\end{aligned}
$$

where $\Omega=\int_{t}^{\infty}\left[\mu_{c}\left\{w L \dot{\tau}_{l}-\pi r \dot{B}_{l}\right\}+\Delta \dot{g}\right] e^{-\theta(s-t)} d s$. Except that distributional considerations are absent here (implying that the distributional characteristics for private consumption and leisure do not appear in equation (39)), there are two important differences between equation (39) and the corre- 
sponding exact welfare measure in Section 3. First, the marginal accounting price of a public good in equation (39) depends on whether the public good is provided by the central government or by the local governments as described in Corollary 2. Second, there are uninternalized external effects implicit in equation (39) - interpreted above - which are summarized by $\Omega$.

\subsection{The Central Government as a Stackelberg Leader}

The strategic interaction among the two levels of government is important for welfare measurement. If the central government does not treat the local public decision variables as exogenous, the principles behind welfare measurement differ from those derived under Nash competition. This is here exemplified by assuming that the central government acts as a Stackelberg leader in the policy problem.

If the central government acts as a Stackelberg leader, it will recognize how the local public decision variables respond to its own policy as well as how the local public costate variables and the local government debt are affected. This means adding equations (26), (28), (29), (30), (31) and (32) to the set of restrictions facing the central government. By using the same notations as in the previous two subsections, the current value Hamiltonian facing the central government takes the form (neglecting the time indicator once again)

$$
\begin{aligned}
H_{c}= & n[v(\bar{w}, \phi)+\Psi(g, G)]+\zeta_{c} \dot{\phi}+\lambda_{c} \dot{K}+\mu_{c} \dot{B}_{c} \\
& +\vartheta_{c} \dot{\zeta}_{l}+\varsigma_{c} \dot{\lambda}_{l}+\iota_{c} \dot{\mu}_{l}+\kappa_{c} \dot{B}_{l}
\end{aligned}
$$

where $\dot{\zeta}_{l}, \dot{\lambda}_{l}$ and $\dot{\mu}_{l}$ are given by equations (30), (31) and (32), respectively, while $\vartheta_{c}, \varsigma_{c}$ and $\iota_{c}$ are the associated costate variables facing the central government. Similarly, $\kappa_{c}$ is the costate variable that the central government attaches to $B_{l}$. The technical details of this problem are, of course, similar to those discussed in the context of the optimization problems described 
above and will, therefore, not be addressed. Let

$$
\left\{\tau_{c}^{\circ}(t), \pi^{\circ}(t), G^{\circ}(t), \tau_{l}^{\circ}(t), g^{\circ}(t)\right\}_{0}^{\infty}
$$

represent the equilibrium of the Stackelberg game (assuming it exists), where $\tau_{l}^{\circ}(t)$ and $g^{\circ}(t)$ for all $t$ are derived by using the reaction functions for $\tau_{l}$ and $g$, given by equations (28) and (29), respectively, when the central government has made its optimal policy choice. Consider the following result;

Proposition 3 If the central government acts as a Stackelberg leader in the policy problem, the national welfare measure can be written $\theta V^{\circ}(t)=H_{c}^{\circ}(t)$.

The proof of Proposition 3 is analogous to the proof of Proposition 2 and is, therefore, omitted. The intuition is that, since the central government acts as a Stackelberg leader and uses the reaction functions and equations of motion from the local decision problems, $\tau_{l}$ and $g$ are not exogenous; instead, they are controlled by the decision variables of the central government. As a consequence, the central government's optimal control problem does not exhibit a nonautonomous time dependence in the same way as in the Nash game. Interestingly, this does not mean that the central government is able to reach the second best resource allocation; only that it can control all time dependent functions (except the utility discount factor) in its optimization problem. In addition, note that Corollary 2 applies, meaning that the procedure for calculating accounting prices for the public goods does not differ between the Nash game and the Stackelberg game.

At the same time, the current value Hamiltonian facing the Stackelberg leader clearly differs from the second best analogue to comprehensive NNP in utility terms defined in Section 3. This is so because the equations of motion for the local costate variables and the local public debt appear in the central government's optimization problem. Therefore, the static equivalent of future utility takes a more complex form here than in the second best, since the central government, in this case, has a broader set of state variables to consider. 


\section{Conclusions}

This paper concerns the public sector in the context of welfare measurement and social accounting. It extends the analyses of previous studies in two main directions. The first is by considering redistribution and public goods in the context of social accounting in a second best economy, where the public revenues are raised by using distortionary taxes. The second is by addressing the federation-like structure that often characterizes the public sector, where the central government and the lower level governments make independent tax and expenditure decisions. I would like to emphasize three broad conclusions;

- The second best analogue to real comprehensive NNP is not in general interpretable in terms of an index comprising only aggregate variables; it also reflects the distribution of private consumption and leisure among consumers. Furthermore, the marginal accounting price of a public good depends on the marginal cost of public funds. As such, to be consistent with welfare accounting in a second best framework, the current practices for social accounting would have to be modified in several important ways.

- The exact welfare measure depends on the way in which the public sector is organized. A federation-like structure, where part of the decisions are made by a central government and part by lower level governments, takes the analysis beyond the second best model. The reason is that public policy may, in this case, give rise to fiscal external effects. Although such external effects have been analyzed in the literature on fiscal federalism, they have been neglected so far in the literature on social accounting. The analysis carried out in the paper explains why fiscal external effects may invalidate Hamiltonian-based welfare measures, as well as shows how fiscal external effects contribute to the exact welfare measure in an economy with several levels of government.

- The exact welfare measure depends on the strategic interaction between the central government and the lower level governments. If the resource allo- 
cation is determined by the outcome of a Nash game between the two levels of government, then the current value Hamiltonian implicit in the central government's optimization problem does not, in general, constitute an exact welfare measure. The reason is that the local public decision variables are, in this case, exogenous to the central government, meaning that the optimal control problem facing the central government becomes nonautonomously time dependent. As a consequence, the welfare contributions of the fiscal external effects must be added to the current value Hamiltonian in order to arrive at an exact welfare measure. If, on the other hand, the central government acts as a Stackelberg leader in the policy problem, it will recognize how the local decision variables respond to its own policy decisions. Therefore, the nonautonomous time dependence mentioned above vanishes, and the current value Hamiltonian facing the central government will become an exact welfare measure.

\section{Appendix}

Derivation of equation (18)

To derive the welfare measure, it is convenient to form the present value Hamiltonian. By neglecting the time indicator for notational convenience, the present value Hamiltonian facing the government is written

$$
\begin{aligned}
H_{p} & =\left[\sum_{i=1}^{n} v\left(\bar{w}, \phi^{i}\right)+n \Phi(G)\right] e^{-\theta t}+\sum_{i=1}^{n} \zeta_{p}^{i} \dot{\phi}^{i}+\lambda_{p} \dot{K}+\mu_{p} \dot{B} \\
& =H_{p}\left(\bar{w}, \bar{r}, G, \phi^{1}, \ldots, \phi^{n}, K, B, \zeta_{p}^{1}, \ldots, \phi_{p}^{n}, \lambda_{p}, \mu_{p}, t\right)
\end{aligned}
$$

where the subindex " $p$ " is used to denote that the Hamiltonian and the costate variables are measured in present value terms. By forming the 
present value Lagrangean, $L_{p}=H_{p}+\nu \bar{r}$, the first order conditions for the control variables can be written

$$
\frac{\partial H_{p}}{\partial \bar{w}}=0, \frac{\partial L_{p}}{\partial \bar{r}}=0 \text { and } \frac{\partial H_{p}}{\partial G}=0
$$

while the costate variables obey

$$
\dot{\zeta}_{p}^{1}=-\frac{\partial H_{p}}{\partial \phi^{1}}, \ldots, \dot{\zeta}_{p}^{n}=-\frac{\partial H_{p}}{\partial \phi^{n}}, \dot{\lambda}_{p}=-\frac{\partial H_{p}}{\partial K} \text { and } \dot{\mu}_{p}=-\frac{\partial H_{p}}{\partial B}
$$

Differentiating the present value Hamiltonian totally with respect to time and using the necessary conditions gives

$$
\begin{aligned}
\frac{d H_{p}}{d t}= & \frac{\partial H_{p}}{\partial \bar{w}} \frac{d \bar{w}}{d t}+\frac{\partial H_{p}}{\partial \bar{r}} \frac{d \bar{r}}{d t}+\frac{\partial H_{p}}{\partial G} \frac{d G}{d t} \\
& +\sum_{i=1}^{n} \frac{\partial H_{p}}{\partial \phi_{p}^{i}} \frac{d \phi_{p}^{i}}{d t}+\frac{\partial H_{p}}{\partial K} \frac{d K}{d t}+\frac{\partial H_{p}}{\partial B} \frac{d B}{d t} \\
& +\sum_{i=1}^{n} \frac{\partial H_{p}}{\partial \zeta_{p}^{i}} \frac{d \zeta_{p}^{i}}{d t}+\frac{\partial H_{p}}{\partial \lambda_{p}} \frac{d \lambda_{p}}{d t}+\frac{\partial H_{p}}{\partial \mu_{p}} \frac{d \mu_{p}}{d t}+\frac{\partial H_{p}}{\partial t} \\
= & \frac{\partial H_{p}}{\partial t}=-\theta\left[\sum_{i=1}^{n} v\left(\bar{w}, \phi^{i}\right)+n \Phi(G)\right]
\end{aligned}
$$

where $\partial H_{p} / \partial \bar{r}=0$ if the nonnegativity constraint does not bind, whereas $d \bar{r} / d t=0$ otherwise. In equation (A3), the second equality comes from using the first order conditions in equations (A1) and (A2), while the third equality is due to the property that $\partial H_{p} / \partial t=-\theta\left[\sum_{i} v\left(\bar{w}, \phi^{i}\right)+n \Phi(G)\right]$ in the second best equilibrium. By solving equation (A3) subject to the transversality condition $\lim _{t \rightarrow \infty} H_{p}(t)=0$, we have

$$
\theta \int_{t}^{\infty}\left[\sum_{i=1}^{n} v\left(\bar{w}^{0}(s), \phi^{i, 0}(s)\right)+n \Phi\left(G^{0}(s)\right)\right] e^{-\theta s} d s=H_{p}^{0}(t)
$$

where the superindex " $0 "$ is used to denote the second best equilibrium. Multiplying equation (A4) by $e^{\theta t}$ gives equation (18).

Proof of Proposition 1 
By using the definition of the instantaneous consumer surplus, we have (neglecting the time indicator)

$$
\sum_{i=1}^{n} u\left(c^{i, 0}, z^{i, 0}\right)+n \Phi\left(G^{0}\right)=\sum_{i=1}^{n} \phi^{i, 0}\left[c^{i, 0}+\bar{w}^{0} z^{i, 0}\right]+n \Phi_{G}\left(G^{0}\right) G^{0}+J^{0}
$$

where the first order conditions for private optimization, $u_{c}\left(c^{i}, z^{i}\right)-\phi^{i}=0$ and $-u_{z}\left(c^{i}, z^{i}\right)+\phi^{i} \bar{w}=0$, have been used. Now,

$$
\sum_{i=1}^{n} \phi^{i, 0}\left[c^{i, 0}+\bar{w}^{0} z^{i, 0}\right]=\phi_{a}^{0}\left[C^{0}+\bar{w}^{0} Z^{0}\right]+n\left[\operatorname{cov}\left(\phi^{0}, c^{0}\right)+\bar{w}^{0} \operatorname{cov}\left(\phi^{0}, z^{0}\right)\right]
$$

Then, using equations (A5) and (A6) together with $\Phi_{G}-(\lambda-\mu)=0$ gives the formula in Proposition 1.

\section{Proof of Proposition 2}

The present value Hamiltonian of the central government's optimization problem is given by (neglecting the time indicator)

$$
\begin{aligned}
H_{c, p} & =n[v(\bar{w}, \phi)+\Psi(g, G)] e^{-\theta t}+\zeta_{c, p} \dot{\phi}+\lambda_{c, p} \dot{K}+\mu_{c, p} \dot{B}_{c} \\
& =H_{p}\left(\bar{w}, \bar{r}, G, \phi, K, B_{c}, \zeta_{c, p}, \lambda_{c, p}, \mu_{c, p}, t\right)
\end{aligned}
$$

where $\zeta_{c, p}=\zeta_{c} e^{-\theta t}$ and similarly for the other shadow prices. Recall that the subindex " $c$ " refers to 'central government' and the subindex " $p$ " to 'present value'. By analogy to the analysis carried out above, the present value Lagrangean is given by $L_{c, p}=H_{c, p}+\nu_{c} \bar{r}$. Note that the taxes decided upon by the central government, $\tau_{c}$ and $\pi$, affect the present value Hamiltonian via $\bar{w}$ and $\bar{r}$. By using $\bar{w}=f_{L}(n l(\bar{w}, \phi), K)\left(1-\tau_{l}-\tau_{c}\right)$ and $\bar{r}=f_{K}(n l(\bar{w}, \phi), K)(1-$ $\pi$ ), we solve for $\bar{w}$ in terms of $\tau_{l}+\tau_{c}, \phi$ and $K$, and solve for $\bar{r}$ in terms of $\bar{w}, \phi, K$ and $\pi$. The first order conditions for the control variables can be written 


$$
\frac{\partial H_{c, p}}{\partial \bar{w}}=0, \frac{\partial L_{c, p}}{\partial \bar{r}}=0 \text { and } \frac{\partial H_{c, p}}{\partial G}=0
$$

whereas the equations of motion for the costate variables obey

$$
\dot{\zeta}_{c, p}=-\frac{\partial H_{c, p}}{\partial \phi}, \dot{\lambda}_{c, p}=-\frac{\partial H_{c, p}}{\partial K} \text { and } \dot{\mu}_{c, p}=-\frac{\partial H_{c, p}}{\partial B_{c}}
$$

Note here that the direct effect of time on the present value Hamiltonian is associated with four variables; the utility discount factor plus the three variables that are exogenous to the central government, $\tau_{l}, B_{l}$ and $g$. By performing the same calculations as those behind equation (A3), we obtain

$$
\begin{aligned}
\frac{d H_{c, p}^{\bullet}}{d t}= & -\theta n\left[v\left(\bar{w}^{\bullet}, \phi^{\bullet}\right)+\Psi\left(g^{\bullet}, G^{\bullet}\right)\right] e^{-\theta t} \\
& +\mu_{c, p}^{\bullet}\left\{w^{\bullet} L^{\bullet} \dot{\tau}_{l}^{\bullet}-\pi^{\bullet} r^{\bullet} \dot{B}_{l}^{\bullet}\right\}+\Delta_{p}^{\bullet} \dot{g}^{\bullet}
\end{aligned}
$$

where $\Delta_{p}=\Delta e^{-\theta t}$. Solving equation (A9) subject to the transversality condition $\lim _{t \rightarrow \infty} H_{c, p}(t)=0$ gives the formula in the proposition.

\section{References}

[1]Aronsson, T. (1998) Welfare Measurement, Green Accounting and Distortionary Taxes. Journal of Public Economics 70, 273-295.

[2]Aronsson, T. and Löfgren, K-G. (1996) Social Accounting and Welfare Measurement in a Growth Model with Human Capital. The Scandinavian Journal of Economics 98, 185-201.

[3]Aronsson, T. and Löfgren, K-G. (1999a) Welfare Equivalent NNP under Distributional Objectives. Economics Letters 63, 239-243.

[4]Aronsson, T. and Löfgren, K-G. (1999b) Pollution Tax Design and Green National Accounting. European Economic Review 43, 1457-1474. 
[5]Aronsson, T., Löfgren, K-G. and Backlund, K. (2004) Welfare Measurement in Imperfect Markets: A Growth Theoretical Approach. Edward Elgar Publishing Limited.

[6]Basar, T. and Olsder, J. (1982) Dynamic Noncooperative Game Theory. Academic Press.

[7]Boadway, R. and Keen, M. (1996). 'Efficiency and the Optimal Direction of Federal-State Transfers', International Tax and Public Finance, 3, $137-55$.

[8]Boadway, R., Marchand, M. and Vigneault, M. (1998). 'The consequences of Overlapping Tax Bases for Redistribution and Public Spending in a Federation', Journal of Public Economics, 68, 453-78.

[9]Chamely, C. (1986) Optimal Taxation of Capital Income in General Equilibrium with Infinite Lives. Econometrica 54, 607-622.

[10]Dahlberg, M. and Lindström, T. (1998) Are Local Governments Governed by Forward Looking Decision Makers? Journal of Urban Economics 44, 254-271.

[11]Dahlby, B. and Wilson, L. (2003) Vertical Fiscal Externalities in a Federation. Journal of Public Economics 87, 917-930.

[12]Deaton, A. (1986) Demand Analysis. In Griliches, Z. and Intriligator, M. Handbook of Econometrics, volume 3, chapter 30, 1767-1839.

[13]Hansson, I. and Stuart, C. (1987). 'The Suboptimality of Local Taxation under Two-Tier Fiscal Federalism', European Journal of Political Economy, 3, 407-411.

[14]Hartwick, J. (1990) Natural Resources, National Accounting and Economic Depreciation. Journal of Public Economics 43, 291-304. 
[15]Hoel, M. (1978) Distribution and Growth as a Differential Game Between Workers and Capitalists. International Economic Review 19, 335-350.

[16]Holtz-Eakin, D, Rosen, H. and Tilly, S. (1994) Intertemporal Analysis of State and Local Government Spending: Theory and Tests. Journal of Urban Economics 35, 159-174.

[17]Johnson, W.R. (1988). 'Income Redistribution in a Federal System', American Economic Review, 78, 570-73.

[18]Keen, M. and Kotsogiannis, C. (2002) Does Federalism Lead to Excessively High Taxes. American Economic Review 92, 363-370.

[19]Lancaster, K. (1973) On the Dynamic Inefficiency of Capitalism. Journal of Political Economy 81, 1092-1109.

[20]Li, C-Z. Löfgren, K-G. (2002) On the Choice of Metrics in Dynamic Welfare Analysis: Utility versus Money Measures. Umeå Economic Studies no 590 .

[21]Michel, P. (1982) On the Transversality Conditions in Infinite Horizon Optimal Control Problems. Econometrica 50, 975-985.

[22]Seierstad, A. and Sydsaeter, K. (1987) Optimal Control Theory with Economic Applications. Amsterdam: North-Holland.

[23] Tovmo, P. (2004) Budget Institutions, Politics and Fiscal Outcomes. Ph D Dissertation, NTNU, Trondheim, Norway.

[24]Weitzman, M.L. (1976) On the Welfare Significance of National product in a Dynamic Economy. The Quarterly Journal of Economics 90, 156162.

[25]Weitzman, M.L. (2001) A Contribution of the Theory of Welfare Accounting. The Scandinavian Journal of Economics 103, 1-24. 
[26]Weitzman, M.L. (2003) Income, Capital, and the Maximum Principle. Harvard University Press: 